\title{
A Computer-Based Product Classification and Component Detection for Demanufacturing Processes
}

\author{
${ }^{1}$ O.E. Simolowo, ${ }^{2}$ A. Mousavi, ${ }^{3}$ P. O. Adjapong \\ 1,2, 3 Department of Systems Engineering, Brunel University, Uxbridge, Middlesex UB8 3PH, UK. \\ ${ }^{2}$ Corresponding Author: esimmar@yahoo.com
}

\begin{abstract}
The aim of this paper is to propose a novel computer based product classification, component detection and tracking for demanufacturing and disassembly process. This is achieved by introducing a series of automated and sequential product scanning, component identification, image analysis, and sorting - leading to development of a Bill of Material (BOM). The produced BOM can then be associated with the relevant disassembly/demanufacture proviso. The proposed integrated image sorting and product classification (ISPC) approach can be considered as a step forward in automation of demanufacturing activities.

The ISPC model proposed in this paper utilises and builds on the state-of-the-art technology and current body of research in Computer Integrated Demanufacturing and Remanufacturing (CIDR). An appraisal of the latest research material and the factors that inhibit CIDR methods in practice are presented. A novel solution for the integration of imaging and material identification techniques to overcome some of the existing shortcomings of automated recycling processes is proposed in this paper.

The proposed product scanning and component detection ISPC software consists of four distinct models: The Repertory Database (RD), the Search Engine (SE), the ProductAttributes Updater (PAU), and the Image Sorting and Classification (ISC) algorithm. The software framework that integrates the four components is presented in this paper. Finally, an overall assessment of applying ISPC at various stages of CIDR processes concludes the article.
\end{abstract}

Key Words: Computer integrated demanufacturing, Disassembly processes, Image-sorting, Product-classification, software-development, recycling technologies, and sustainable manufacture 


\section{Introduction}

CIDR involves the extension of the elements of computer integrated manufacturing (CIM) to recycling and remanufacturing operations for optimum materials recovery. Image analysis entails the acquisition of information about an object under study through signal processing of which the input is an image of the object such as scanned photo, digital photograph or frame of video and the output is a set of representative characteristics or parameters of the analyzed image. An integration of these two defined processes for optimum material recovery of used products is the main focus of this research.

The purpose of this research article is to report on a novel technique to enhance the automation of recycling processes. The enhancement is achieved by integrating image processing techniques to conduct an end-of-life (EOL) product-content detection and product disassembly analysis. In this paper we report on the development of repertory software capable of storing and linking product content information from manufactures to demanufacturers. The purpose for this connection is the ability to compare the original components of the artefact with that of the EOL one. The image correlation between original component and the EOL will reduce image and component identification process.

The software further uses the captured information coupled with acquired scanned and digital images to perform image sorting and classification of the used product so as to determine the next demanufacturing path.

The proposed detection and component identification process will contribute to the more ambitious future full automation of product recycling processes. The information produced can then be used to continuously monitor and measure the environmental impact of recycled and re-usable products and the industry as whole. This information can help with measuring the levels of pollution caused by waste disposal and material/component recovery during recycling processes.

\section{Background Literature on Research Inhibitions and Advantages of ISPC}

The proposed ISPC system suggests the use of appropriate X-ray imaging equipment (such as scanners) to reduce reliance on manual inspection of disposed products for recycling. An automated scanning and component identification technology will significantly reduce the cost and time of the demanufacturing or disassembly process . X-ray imaging techniques include Plain Radiographs (X-ray), Magnetic Resonance Imaging (MRI), Computed tomography (CT), Ultrasound (US), Fluoroscopy and Nuclear Medicine (NM). More recent 
X-ray imaging methods such as the imaging techniques with Synchrotron Radiation (ITSR) (Cyril 2009) and the Ultrasound-modulated optical tomography (UMOT) (Gang 2005) are among those in use today. The latter combines unique optical contrast, good spatial resolution, and large imaging depth in its features. In updating the repertory database of the ISPC software the MRI (Simon 2006) or CT techniques are suitable for image capturing. In addition, a more useful approach that could be applied in this work is the third generation of automated X-ray image processing. These image processing platforms are specifically designed to detect shapes, position and presence of components using Neural Network Techniques.

To date several methods have been proposed to overcome the challenges of automation and computer integrated demanufacturing for recycling purposes (Williams 2007, and Tianyang et al. 2006). These methods have been restricted by a number of factors that has resulted into an explosion of solutions by researchers and practitioners. The scope of this paper is limited to the automation of product scanning, image analysis and creation of a component data module that can be used for downstream recycling and demanufacturing operations. However, due to the nature of the study our literature review covers the full EOL cycle of a product, encompassing the detection, sorting, data management, and disassembly/demanufacturing processes. Earlier solutions for demanufacturing processes introduced material information overture that were suggested by Scheidt and Zong (1994), Klausner (1998), Seliger et al (2001). The solution focused on obtaining and maintaining product information from the original component manufacturers and transferring the information to third-party recyclers. Even though practical, this method created complications for the recycling industry as time past. The complications were due to products undergoing regular changes in design and type of material used in their components and maintaining an updated set of information was expensive and at times confusing leading to extra labourer time during the demanufacturing or disassembly process. The proposed ISPC solution in this paper tends to address this shortcoming by incorporating the information about the state of the product during its design and production phase. It will then link this information to the information of the disposed product at its EOL stage. The operators at the recycling centre will then be able to compare the current product with the latest information obtained from the point of manufacturing to EOL. This procedure eliminates the need for information link and problem posed by product change. A detailed discussion of this procedure is presented in section 3 of this paper. 
Willaims (2007) extracted the procedures for generating demanufacturing sequences from earlier works carried out by researchers for example; Gungor and Gupta (1998), Zussman and Zhou (1999), Srinivasan et al. (1999), Srinivasan and Gadh (2000), Zha and Lim (2000), Dini et al. (2001), Erdos et al. (2001), Das et al. (2003), and Singh et al. (2003).. These include product computer-aided design (CAD) file, product BOM, part mating data, material content, value of recovered materials, product topology and precedence relations for a product. Murphy et al (2001) suggested the necessity of integrating decision making for pre-sorting and other processes to achieve the most effective material recovery system. Many set backs are experienced in designing robust automated systems due to random change in the condition of recyclable/reusable products. These conditions may include: physical damage, rust, missing components and replaced components. Therefore, for effective detection and product sorting process, there is a need for a cost effective and suitable automated visual analysis. This can be realised by installing scanning and image processing technology that provides information about the state of the product as well as an account of the hidden parts (i.e. undetectable parts by naked eye or photography) it contains. The automated product scanning and the BOM produced should reduce the costs of disassembly, labour time, and handling of hazardous substances.

Our proposed ISPC has also interrogated the reverse supply chain literature about demand-driven selective disassembly planning by Williams (2007) and other works on selective and optimal disassembly planning in demanufacturing by Chung and Peng (2006) and Grochowski and Tang (2009). The novel image analysis software for the purpose of creating a disassembly process plan proposed in this paper builds on and improves on the suggestions given by reverse supply chain literature. It has the capability to carry out image sorting and classification of products based on component contents as dictated by changes undergone by the product during life use. This procedure will provide the needed advancerepertory of product attributes as pointed out by Williams J.A.S. (2007).

In their work on disassembly planning of mechanical systems using the Generic Algorithm Based (GAB), Fabio and Giovanna (2007) stressed the need for obtaining detailed information on significant characteristics of recyclable/reusable product components. In order to successfully implement the GAB for the selective-disassembly and partial/complete disassembly, the detailed information on components is essential. Moreover, the requirement for expert Petri net approaches for selective robotic disassembly have been stated by William (2007) to include new ways of integrating imaging and materials identification techniques in CIDR. Even though the present work described in this paper does not provide a complete 
solution to this requirement, however it presents a foundational novel approach that can be used to supply vital product information that are needed to generate optimal automated disassembly plans.

On the other hand research on cost analysis of disassembly operations are continually carried out to assist demanufacturers and manufacturers meet economical and environmental demands. Two important considerations in the development of cost-effective disassembly schedules as presented by Tang et al. (2002) are (i) what should be the extent of dissembling a product (Meacham 1999, Zussman and Zhou 1999, 2000, Lambert 1997, 2002) (ii) ways of determining cost-effective disassembly (Zussman and Zhou 1998, Dutta and Woo 1995, Woo and Dutta 1991, Srinivasan and Gadh 1998a, b). Image sorting which involves productcontent analysis and product classification prior to disassembly operations will eliminate unnecessary labour, cost and other automation drudgery due to on-the-spot knowledge of the material-content of the EOL product, thereby enhancing proper planning for cost-effective disassembly processes.

Tianyang Dong et al. (2006) cited another method to implement Petri net algorithm to enhance recycling automation while discussing a novel approach towards the automatic generation of disassembly sequence from hierarchical attributed graph (HALG) representation and the consideration for subassemblies. The Petri net approach proposes the employment of an algorithm that automatically generates a disassembly Petri net (DPN) that can be analysed to generate all feasible disassembly process plans and to determine the optimal disassembly process plans also putting cost functions into consideration ( Kendra E. M. et al. 2001). Other works that focused on the complexities of this approach, the generation of low-level expert Petri net and the integration of this approach to obtain flexible demanufacturing systems show that this method has been inhibited by the lack of available EOL product content information (Das et al. 2003, , Singh et al. 2003, and Ranky et al. 2003).

Kopacek and Kopacek (2006) employed the use of compatible hardware and software models that included image processing systems among other tools in the development of a modular system for creating flexible, intelligent, "low cost" disassembly cells for printed circuit boards (PCBs). Williams (2006) highlighted the reliance of automated disassembly on sensors and algorithms for image processing, grippers for product handling, a decision tool for disassembly task planning, and robotic tooling (inspired by Weigl et al (1996)). Jianjun et al (2008) discussed four benefits of achieving efficient disassembly of products as opposed to recycling a product by shredding. In summary, they are: (i) possibility of reusing or 
refurbishing components of adequate quality (ii) categorization of metallic parts towards increasing their recycling value (iii) easy removal of plastic parts for recycling, and (iv) separation of hazardous materials. Research activities for improving the latter method are on going. These include the modelling of post-fragment waste stream with shredder facilities (Gareth and Shahin 2009) and other similar works (Van and Reuter 2006, Van et al 2005). The main focus of these works (Gareth and Shahin 2009) is the development of future dynamic shredding facilities of which part of the EOL product information is needed. We believe that the information can be obtained by integrating the shredding equipments with the proposed ISPC, leading to more cost effective and efficient shredding technology.

\section{Integrating Image Sorting and Product Classification}

The approach reported in this paper focuses on integrating imaging techniques at the early stages of computer integrated demanufacturing and remanufacturing (CIDR) procedures. The repertory software developed will be capable of carrying out productcontent-analysis, pre-sorting, pre-classification of products and generation of material content information to be communicated with disassembly sequence planning. The automatic generation of disassembly sequence (e.g. Hu et al. 2002, Desai and Mital 2003, Torres et al. 2003, Kuo Tsai C. 2000, Kendra et al. 2001) is outside the scope of this paper. . But it will help in dealing with some of the factors that may limit the full implementation of disassembly methods and other advance computer integrated demanufacturing and remanufacturing technologies that rely on detail product and component information.

The software structure shown in Figure 1 illustrates the overall structure of the image sorting and product classification (ISPC) software.

\section{INSERT FIGURE 1}

The ISPC software comprises $\mathrm{M}_{1}, \mathrm{M}_{2}, \mathrm{M}_{3}$ and $\mathrm{M}_{4}$ modules that contain the algorithms that perform the functions of (i) data base formation of the base product, (ii) product information search,(iii)updates for new products and (iv) image sorting and classification analysis of EOL products respectively. Modules $\mathbf{M}_{1 \mathrm{a}}, \mathbf{M}_{1 \mathrm{~b}}$ and $\mathbf{M}_{1 \mathbf{a b 1}}$ of $\mathbf{M}_{1}$ handle the storage and processing of product attributes like product name, manufacturer, year of manufacture, specification number among other vital information (such as geometric attributes or specially built-in identification attributes for this purpose) for the product-assembly and components. 
Scanned images of products are obtained using appropriate scanners by ISPC operators at EOL. Digital images are also taken at the Beginning of Life (BOL) and loaded on the ISPC software database. The information provided by the two images will be used for comparing and identifying components for the demanufacturing and disassembly process.

To overcome the problem of missing labels, stickers and parts that identify the products at EOL, the digital image (regardless of its colour) combined with the geometric attributes of the products (such as size, weight range etc.) that were stored in the ISPC software database at (BOL) will assist in product recognition at EOL. The process of uploading and filing the scanned and digital images of the base products and components in the data base are also carried out by $\mathrm{M}_{1 \mathrm{ab} 2}$. The search of information about images and product attributes of the EOL items are performed by $\mathrm{M}_{2 \mathrm{a}}$ and $\mathrm{M}_{2 \mathrm{~b}}$ respectively. The software also makes it possible to continually update its database with attributes $\left(\mathrm{M}_{3 \mathrm{ab} 1}\right)$ and images $\left(\mathrm{M}_{3 \mathrm{ab} 2}\right)$ of new products thereby constantly expanding its application. The functions of image sorting and classification are represented by modules $\mathrm{M}_{4 \mathrm{a}}$ and $\mathrm{M}_{4 \mathrm{~b}}$ respectively.

The integration of imaging techniques like ISPC into the automation of demanufacturing processes as reviewed in this paper has been shown as areas of present and further works (Jorgensen and Anderson 1996, Williams 2007). ISPC is a new approach to the proposed "future centralised or decentralised information flow for product environment profile" as proposed in many research (Williams 2007, Peng et al. 2003, Scheidt and Zong 1994, Spath 1994, Zeid et al. 1996, Klaussner et al. 1998, Seliger et al. 2001, Grenchus et al. 1998, Kuo et al. 2000, Chung et al. 2003, Das et al. 2003.). It is therefore seen that the acquired product data will enhance demanufacturing planning for reuse or recycling.

Figure 2 is a representation of the processes involved in the integration of ISPC in demanufacturing using the software modules described in figure 1.

\section{INSERT FIGURE 2}

From Figure 2, product-state information can flow between the ISPC software and the five players: (i) manufacturers (ii) retailers (iii) users, (iv) collectors of EOL products, and (v) the demanufacturers. The two most important stages involved in the integration of ISPC depicted in Figure 2 are here in discussed. 
(i) Database build-up $\left(M_{1}\right)$ : The attribute, scanned image, external component design details and other information required for identifying the product assembly $\left(\mathrm{M}_{1 \mathrm{a}}\right)$ and product components $\left(\mathrm{M}_{1 \mathrm{~b}}\right)$ at EOL are obtained at manufacturing point i.e. beginning of life (BOL) of product by operators in the ISPC units.

(ii) Image sorting and item classification $\left(M_{4}\right)$ : At EOL the scanned images of the products are obtained by the ISPC units of the demanufacturers. These images are compared with those of the ISPC software obtained from the manufacturers in step (i). The comparison is completed after the product is searched $\left(\mathrm{M}_{3}\right)$ and identified within the software database using the stored BOL information and the physical EOL attributes. Image sorting and product classification are performed thereafter.

There are benefits derived from other processes that constitute the integration of ISPC in demanufacturing also shown in Figure 2. These processes and their benefits include:

(a) Content analysis for new products by retailers: The installation of appropriate ISPC units (comprising software for selected products and appropriate scanners) at retail centres will provide product-state checks for items purchased. The scanned images of the newly purchased products obtained at these retail centres will be compared and analysed with their base images obtained from manufacturers at BOL thereby eliminate products with production faults

(b) Content analysis for used products by buyers: Following the same procedures as discussed in (a), buyers of used products can perform content analysis of bought items at retail or independent ISPC centres that have database for such items.

(c) Pre-sorting at collection centres: ISPC units set up at collection centres for preferred products like electronics will perform primary pre-sorting and pre-classification (following steps $a$ and $b$ ) before getting to the demanufacturers. This will help streamline the unwanted products in the process line of disassembly thereby enabling the advanced reverse-supply needed for selective or demand-driven disassembly planning. Also from Figure 2, the actual ISPC analysis is carried out at demanufacturing points by making use of stored information, the newly obtained EOL product details and the functions available in modules $\mathrm{M}_{2}$ and $\mathrm{M}_{4}$.

\section{The ISPC Software}

In Figure 3 the component-algorithms that describe the complete flow of operation of the ISPC software are shown.

\section{INSERT FIGURE 3}


The MATLAB programming codes comprising of the image processing module and the graphical user interface (GUI) tools that were used in the development of this application were embedded in the Graphical User Interface Development Environment (GUIDE). Future works may interface the present program with other compatible high level languages like the Visual $\mathrm{C}++$ or $\mathrm{C}^{\#}$ in order to improve and extend its application and usability. The function of the ISPC software is described based on the two main processes its name suggests namely; (i) Image sorting and (ii) Product classification.

The Image sorting capability of the software entails the use of the database of scanned digital images and the associated base product attributes obtained from source (i.e. manufacturer or distributors) at the beginning-of-life (BOF) of the product (i.e. new product). This attribute is compared with that captured from the EOL product (or any product to be recycled) to determine the complete information on its design, structure and material content. The principle of image subtraction has been employed to determine the content and state of the product at the EOL.

Figure 4 shows the image sorting analysis for a cell phone using image subtraction principles (Mathworks 2009). The two uploaded scanned images represent the base and EOL products and the empty dark shade appearing in the middle of the two images is the result of the sorting and image subtraction.

\section{INSERT FIGURE 4}

From figure 4 one can observe that the content of the artefact (in this case a phone) at its EOL is exactly as it was when newly manufactured, i.e. with no internal damage. A high level of precision is required when placing the products for image scanning and analysis as seen in Figure 4 and 7. This precision positioning is not necessary for digital images that are needed only for identification purposes. Information on precision setting are to be contained in the "teach" section of the ISPC software depending on the type of scanner used. The information obtained from the sorting analysis on every product includes the following:

(i) Percentage and number of parts present (\%PP) and their bill of materials.

(ii) Percentage and number of parts missing (\%PM) and their bill of materials.

(iii) Percentage and number of parts damaged (\%PD) and their bill of materials. 
The Product classification function of the software depends on the output of the image sorting procedure. This procedure is carried out on each item. The product is subsequently classified as: (i) reusable, after minor or major servicing, (ii) repairable, where few parts need replacement depending on the product analysed, (iii) recyclable, considering either the whole or parts of the product, and (iv) disposable, where product is extensively damaged or extremely hazardous beyond use.

The result of the analysis in Figure 4 shows that $\mathrm{PP}=100 \%, \mathrm{PM}=0 \%$ and $\mathrm{PD}=0 \%$. These figures imply that the product is reusable after minor servicing. The product classification stage also enables the skilled user (operator) of the software to give expertise additional information about the product and its components post analysis, and prepare the product for demanufacturing process. The operational-flow algorithm shown in Figure 3 consists of three steps to perform four broad functions and other sub functions. The first part of the program flow consists of algorithm that make up modules $\mathrm{M}_{1}$ and $\mathrm{M}_{3}$ which can be called up at different stages as required and are used for initial data base build-up and updates respectively. The software builds up its database with information of any product fed into it and of all replaceable components or parts that make up the product. The BOM generated by the software are for both individual components as well as for the product assembly. This is to assist in recognising parts needing replacement and to determine paths to follow in disassembling planning. The second group of algorithm which constitutes the $\mathrm{M}_{3}$ module is critical in ISPC procedure because at this stage the stored information of the exact base product corresponding to the product to be analysed is brought to view from attributes of similar products using the search engine algorithms. The algorithm for the search engine is such that any of the red attribute that is associated with the product can be used to search for the corresponding base product in the repertory files of the software to cross check the search results. The third part of the overall algorithm shown in Figure 4 as dotted lines performs the image sorting and classification process once the exact base product information required has been obtained from data base.

An overview of the interfaces of the ISPC software being developed is captured by the screenshots of Figures 5 - 7. The configuration of the input interface shown in Figure 5 is generated by GUI controls contained in modules $\mathrm{M}_{1 \mathrm{a}}$ and $\mathrm{M}_{1 \mathrm{~b}}$ (reference to Figure 1). The attributes of the product-assembly and its components are fed into the ISPC data base through the input interface.

\section{INSERT FIGURE 5}


These GUI controls are also used for updating or modifying product-assemblies attributes $\left(\mathrm{M}_{3 \mathrm{a}}\right)$ and their components $\left(\mathrm{M}_{3 \mathrm{~b}}\right)$. The other graphical displays shown in Figure 5 include the browser window to enable proper filing and storage of images, the display controls for modules $\mathrm{M}_{2 \mathrm{a}}$ and $\mathrm{M}_{2 \mathrm{~b}}$ that constitute the search engine for images and attributes respectively, the user interfaces controls for modules $\mathrm{M}_{4 \mathrm{a}}$ and $\mathrm{M}_{4 \mathrm{~b}}$ that perform the sorting and classification analysis respectively. In Figure 6, the digital and scanned images of a toaster stored in the database are uploaded for presentation. The images are at different orientations because there is no content analysis between a scanned image and a digital picture. In Figure 7 the representations of the analysed product is displayed.

\section{INSERT FIGURES 6 AND 7}

\section{Future Research and Application of ISPC}

At present, the ISPC software applies the principle of image subtraction (Mathworks 2009) for sorting and classifying products based on material-content analysis at their EOL. The application of Neural Networks in image processing that enables the detection of foreign objects, shape defects, absence or excess of products, overly compacted products, object position, missing items, and item count (even if parts overlay) are areas being researched in obtaining precise number of damaged or missing parts. Future application of ISPC could be extended to disassembly sequence planning. Shown in Table 1 (see appendix), a summary of the present advantages and future role of ISPC in demanufacturing is presented. It lists the procedures that were employed to improve CIDR processes. The processes include the disassembly models (e.g. component connections and mating) using Direct Graph and AND/OR graph (Tang et al. 2002). All these models and methods considered have some constraints that could be overcome by the future application of ISPC technique. In a work carried out on the modelling, planning and application of disassembly processes (Tang et al. 2002), the trend in research activities on disassembly procedure was reported. In specific, the concluding remarks were about generation of predictive optimal sequences with reference to known information about the product assembly. However, in practical situations, the state of some of the products to be disassembled is unknown. It therefore becomes imperative to focus future research works on procedures that increases the level of EOL product-content 
predictability thereby enhancing the building of responsive dynamic disassembly systems such as real-time disassembly systems.

Future research on the combination of ISPC data and other EOL feature information such as $\mathrm{CAD}$ files, 3-D models, mating and precedence relations would enhance the heuristic method as well as other methods that have been used to improve disassembly efficiency over the years. It will also result in selective disassembly planning which will involve:

(i) Elimination of unnecessary disassembly sequences

(ii) Categorization disassembly sequences with similar EOL material content. The integration of ISPC in disassembly sequence planning for the product-assembly shown in Figure 8(a) with its interference matrix and the disassembly sequence shown in Figures 8 (b) and (c) respectively is explained in Figure 9. The three stages involved in the integration are:

(i) A comprehensive acquisition of disassembly bill of materials (DBOM)

(ii) Selective generation of disassembly sequences

(iii) Creation of selective task work stations

\section{INSERT FIGURES 8(a-c) AND 9}

The integration of data generated by ISPC software with other DBOM at stage 1 helps in the subsequent categorization of the disassembly sequences at stage 2 . Tools and workstation for destructive and non-destructive disassembling processes can be devised at plant layout stage with special attention to possibly use cellular manufacturing layout. Unnecessary operations and sequence generation would be eliminated for cases where the ISPC detection shows EOL has no missing or damaged component $(\% \mathrm{PP}=100)$. The sequence generation can also be reduced for EOL products needing minor servicing before putting them into reuse. In a situation of extensive damage and loss of EOL Product components, the determination of the type of destructive and non-destructive processes is also aided. In short, using the proposed system has one direct and one indirect advantage. The direct advantage is that it significantly reduces the time for detection, classification and sorting process. The indirect advantage is that it contributes to reducing the time and effort for disassembly process by categorising products and directing material to dedicated stations, thus making recycling-reusing of manufactured products less labour intensive. 


\section{Conclusion}

Various researchers have stressed the need for new approaches to integrate imaging, materials identification methods, decision making for collection, pre-sorting and other processes of demanufacturing to produce optimum material recovery systems. This paper has reviewed some of the models and methods that have advanced computer integrated demanufacturing and remanufacturing (CIDR) in recent years. Building on recent body of knowledge this paper introduces a novel software tool for the integration of image processing and product classification in CIDR processes. It offers a solution for automating the component detection and product classification process that demanufacturing operations require prior to disassembly of product at the end of their life cycle.

The constraints of some of the existing methods that have delayed the advancement and proliferation of demanufacturing processes were discussed. Those constraints were discussed with respect to the methods for managing product materials information, demanddriven selective disassembly planning, logic-reverse assembly strategies and other disassembly process models. Moreover, in this paper we highlighted the current shortcomings in linking the information between manufacturers and demanufacturers. In specific, the uncertainty of a products journey in its life cycle to the time it reaches its EOL were explained; and how the proposed Image Sorting and Product Classification (ISPC) software tool may contribute to providing the linkage. Although at this stage the ISPC solution only deals with the product identification and information management, but it builds the first building block for an integrated automatic disassembly process planning and optimisation.

The ability of the ISPC repertory software to preserve the information link by acquiring all necessary product-content attributes from manufactures, store it and later employ it in the product analysis at the point of demanufacturing by recyclers regardless of the changes the product might have undergone gives it an important role in enhancing various approaches in CIDR and also in future development of multifunctional robot systems when combined with other EOL product feature information such as CAD files, mating patterns, precedence relations and topology

\section{Acknowledgement}

This research which was carried out at School of Engineering and Design Brunel University, West London and was supported by the University of Ibadan MacArthur Foundation Grant. 
The images used for software validation and development were those of Prof. Satre Stuelke founder of the Radiology Art project in New York

\section{References}

Chung, C. and Peng, Q., 2006. Evolutionary sequence planning for selective disassembly in de-manufacturing. International Journal of Computer Integrated Manufacturing, 19, 278286

Chung, J., Koh, Y., Lee, H. and Hur, T., 2003. Web based ecodesign supporting system for electronic products. Proceedings of IEEE International Symposium on Electronics and the Environment, Boston, MA, 14, 246-250.

Cyril, P. and Hugh, J., 2009. The First International Workshop on Imaging Techniques with Synchrotron Radiation, Synchrotron Radiation News, 22, (3), 39 - 40

Das, S., Yedlarajiah, D. and Gurram, S., DISX: 2003. A procedure for the automated generation of disassembly process plans. Proceedings of the IEEE International Symposium on Electronics and the Environment, Boston, MA, 12-14, 60-65.

Desai, A. and Mital, A., 2003. Evaluation of disassemblability to enable design for disassembly in mass production. Internationa Journal of Industrial Ergonomics, 32, 265281.

Dini, G., Failli, F. and Santochi, M., 2001. A disassembly planning software system for the optimization of recycling processes. Production Planning and Control, 12, 2-12.

Dutta, D. and Woo, T.C., 1995. Algorithm for multiple disassembly and parallel assemblies. Journal of Engineering for Industry, Transactions. of ASME, 117, 102-109

Erdos, G., Kis, T. and Xirouchakis, P., 2001. Modelling and evaluating product end-of-life options. International Journal of Production Research, 39, 1203-1220.

Fabio, G. and Giovanna, F., 2007. Disassembly planning of mechanical systems for service and recovery: genetic algorithms based approach. Journal of Intelligent Manufacturing,18, 313-329

Gareth, C. and Shahin, R., 2009. Modelling of post-fragmentation waste stream processing within UK shredder facilities. Waste Management, 29, 44-53

Gang, Y., 2005. Ultrasound Modulated Optical Tomography for Tissue Imaging. Encyclopedia of Agricultural, Food, and Biological Engineering.

Grenchus, E., Keene, R., Nobs, C., Brinkley, A., Kirby, J.R., Pitts, D. and Wadehra, I., 1998. Linking demanufacturing operations with product DFE initiatives. Proceedings of the IEEE International Symposium on Electronics and the Environment, Oakbrook, IL, 4-6, 270-274. 
Grochowski, D. and Tang, Y., 2009. A machine learning approach for optimal disassembly planning. International Journal of Computer Integrated Manufacturing, 22, 4, 374 - 383

Gungor, A., and Gupta, S.M., 1998. Disassembly sequence planning for products with defective parts in product recovery, Computers and Industrial Engineering, 1-2, 161-164.

Hu, D., Hu, Y. and Li, C., 2002. Mechanical product disassembly sequence and path planning based on knowledge and geometric reasoning. International Journal of Advanced Manufacturing Technology, 2002, 19, 688-696.

Jianjun, Y., Bin, Y., Lei, D., Chenggang, L. and Diqing, H., 2007 Research on the selective disassembly strategy of mechanical parts based on the generalized CAD model. International Journal of Advanced Manufacturing Technology, 37, 599-604.

Jorgensen, T.M. and Andersen, A., W., 1996. Shape recognition system for automatic disassembly of TV-sets. Proceedings Of IEEE International Conference on Image Processing Lausanne, Switzerland, 2, 653-656.

Kendra, E. M., Gungor, A. and Gupta, S.M, 2001. Petri net approach to disassembly process planning for products with complex AND/OR precedence relationships. European Journal of Operational Research, 135, 428-449.

Klausner, M., Grimm, W.M. and Hendrickson, C., 1998. Reuse of electric motors in consumer products. Journal of Industrial Ecology, 2, 89-102.

Kopacek, P. and Kopacek, B., 2006. Intelligent, flexible disassembly. International Journal of Advanced Manufacturing Technology, 30, 5-6, 554-560.

Kuo Tsai, C., 2000. Disassembly sequence and cost analysis for electromechanical products. Robotics and Computer Integrated Manufacturing, 16, 43-54.

Lambert, A.J.D., 1997. Optimal disassembly of complex products. International Journal of Production Research, 35, 9, 2509-2523.

Lambert, A.J.D. and Gupta, S.M., 2002. Demand-driven disassembly optimization for electronic products. Journal of Electronic Manufacturing, 11, 121-135.

Matlab®, 2009. The Mathworks Inc., 1984-2009 (Image processingToolbox).

Meacham, A., Uzzoy, R. and Venkatadri, U., 1999. Optimal disassembly configuration for single and multiple products. Journal of Manufacturing Systems, 18, 5, 311-322.

Murphy, C.F., Dillon, P.S. and Pitts, G. E., 2001. Economic and logistical modeling for regional processing and recovery of engineering thermoplastics. Proceedings of the IEEE Symposium on Electronics and the Environment, 229-35.

Peng, M., Guanghong, D., Dong, X., Xeuping, L., Ying, W., Fangyi, L. and Yi, L., 2003. An information system management of assessment of disassembly and recycle. Proceedings of 
the IEEE International Symposium on Electronics and the Environment, Boston, MA, 12-14 286-290.

Ranky, P.G., Subramanyam, M., Caudill, R.J., Limaye, K. and Alli, N., 2003. Dynamic scheduling and line balancing methods, and software tools for lean and reconfigurable disassembly cells and lines. Proceedings of the IEEE International Symposium on Electronics and the Environment, Boston, MA, 12-14, 234-239.

Seliger, G., Basdere, B. and Keil, T., 2001. E-cycling platform for profitable reuse. Proceedings of the IEEE International Symposium on assembly and Task Planning, Fukuoka, Japan, 453-457.

Scheidt, L.-G. and Zong, S., 1994. An approach to achieve reusability of electronic modules. Proceedings of the IEEE International Symposium on Electronics and the Environment, San Francisco, CA, 2-4, 331-336.

Simon, J., 2006. Doran Magnetic Resonance Imaging (MRI). Encyclopedia of Biomaterials and Biomedical Engineering.

Singh, A.K., Tiwari, M.K. and Mukhopadhyay, S.K., 2003. Modelling and planning of the disassembly processes using an enhanced expert Petri net. International Journal of Production Research, 41, 3761-3792.

Spath, D., 1994. The utilization of hypermedia-based information systems for developing recyclable products and for disassembly planning. Annals of CIRP, 43, 153-156.

Srinivasan, H. and Gadh, R., 1998a. Ageometric algorithm for single selective disassembly using the wave propagation abstraction. Computer-Aided Design, 30 (8), 603-613.

Srinivasan, H. and Gadh, R., 1998b. Complexity reduction in geometric selective disassembly using the wave propagation abstraction. Proceedings of the International Conference on Robotics \& Automation. Leuven, Belgium, 1478-1483.

Srinivasan, H., Figueroa, R. and Gadh, R., 1999. Selective disassembly for virtual prototyping as applied to de-manufacturing. Robotics and Computer Integrated Manufacturing, 15, 231-245.

Srinivasan, H. and Gadh, R., 2000. Efficient geometric disassembly of multiple components from an assembly using wave propagation. Journal of Mechanical Design, 122, 179-184.

Steinhilper, R., 1998. Remanufacturing: the Ultimate Form of Recycling, (Fraunhofer IRB Verlag: Stuttgart).

Tang, Y., Zhou, M., Zussman, E., and Caudill, R., 2002. Disassembly modelling, Planning and Application. Journal of Manufacturing Systems, 21, 3, 200-217. 
Tianyang, D., Ling, Z., Ruofeng, T. and Jinxiang, D., 2006. A hierarchical approach to disassembly sequence planning for mechanical product. International Journal of Advanced Manufacturing Technology,30, 507-520

Torres, F., Puente, S. T. and Aracil, R., 2003. Disassembly planning based on precedence relations among assemblies. International Journal of Advanced Manufacturing Technology, 21, 317-327.

Van, S. and Reuter, M., 2006. Modelling of liberation in recycling passenger vehicles, Proceedings of the XIII International Minerals Processing Congress, 3, 3-8.

Van, S., Reuter, M. and Richard, A., 2005. A comparison of the modeling and liberation in minerals processing and shredding of passenger vehicles. In: Schlesinger, M.E. (Ed.), EPD Congress. TMS (The Minerals, Metals \& Materials Society), 1039-1052.

Weigl A., Hohm K. and Tolle H., 1996. A flexible tactile grasping strategy for automated robotic disassembly. $27^{\text {th }}$ International Symposium on Industrial Robots,

Williams, J.A.S., 2006. A review of electronics demanufacturing processes. Resources, Conservation and Recycling, 47 195-208

Williams, J.A.S., 2007. A review of research towards computer integrated demanufacturing for materials recovery. International Journal of Computer Integrated Manufacturing, 20 (8), $773-780$

Woo, T.C. and Dutta, D., 1991. Automatic disassembly and total ordering in three dimensions. Journal of Engineering for Industry, Transactions of ASME. 113 (6), 207-213.

Zha, X.F. and Lim, S.Y.E., 2000. Assembly/disassembly task planning and simulation using expert Petri nets. International Journal of Production Research, 38, 3639-3676.

Zeid, I., Gupta, S.M. and Bardasz, T., 1996. A case-based reasoning approach to planning for disassembly. Journal of Intelligent Manufacturing, 8, 97-106.

Zussman, E. and Zhou, M. and Caudill, R., 1998. Disassembly Petri Net approach to modelling and planning disassembly processes of electronic products. Proceedings of IEEE International Symposium On Electronics and Environment, Oak Brook. IL, 331-337.

Zussman, E. and Zhou, M., A 1999. methodology for modelling and adaptive planning of disassembly processes. IEEE Trans. Robotics Automn,15, 190-194.

Zussman, E. and Zhou, M., 2000. Design and implementation of an adaptive process planner for disassembly processes. IEEE Trans. Robotics Autumn, 16, (2), 171-179. 
Appendix

INSERT TABLE 1 